\title{
Flat lensing by periodic loss-modulated materials
}

\author{
N. Kumar, ${ }^{1}$ R. Herrero, ${ }^{1}$ M. Botey, ${ }^{2, *}$ and K. Staliunas ${ }^{1,3}$ \\ ${ }^{1}$ Department de Fisica i Enginyeria Nuclear, Universitat Politècnica de Catalunya, Colom 11, 08222 Terrassa, Spain \\ ${ }^{2}$ Department de Fisica i Enginyeria Nuclear, Universitat Politècnica de Catalunya, Urgell 187, 08036 Barcelona, Spain \\ ${ }^{3}$ Instituciò Catalana de Recerca i Estudis Advançats (ICREA), Pg.Lluis Companys, 23, 08010 Barcelona, Spain \\ *Corresponding author: muriel.botey@upc.edu
}

Received June 7, 2013; revised July 24, 2013; accepted August 10, 2013; posted August 12, 2013 (Doc. ID 191858); published September 23, 2013

We propose a flat lensing effect using a periodic loss-modulated material. In particular, we consider a twodimensional square and rhombic arrangement of lossy cylinders embedded in a host media with the same refractive index. The effect is predicted by the dispersion curves obtained by a coupled mode expansion of Maxwell equations and by numerical beam propagation experiments. From both analytical and numerical studies, we show that, for a range of frequencies, light beams undergo negative diffraction on propagation through the loss-modulated medium, providing a window of high transmission. The phase shifts accumulated by negative diffraction within the structure are then compensated by normal diffraction, leading to substantial focalization beyond it. (c) 2013 Optical Society of America

OCIS codes: $\quad$ (160.4670) Optical materials; (260.1960) Diffraction theory; (290.5830) Scattering, Brillouin;

(160.3918) Metamaterials; (160.5298) Photonic crystals.

http://dx.doi.org/10.1364/JOSAB.30.002684

\section{INTRODUCTION}

Materials with spatially modulated refractive index-photonic crystals (PhCs) - have shown the ability to manage the dispersion of light. The control over light propagation is twofold: on one hand, the modification of the dispersion relations in the frequency domain results in temporal effects, such as photonic bandgaps [1,2] or slowing light down; on the other, the modification of the spatial dispersion enables managing diffraction and provides associated beam propagation effects. As an example, self-collimation [3-5] , focalization and imaging beyond a structure with a flat-flat interface (flat PhC lensing) [6-8 $]$, and spatial (angular) filtering [ $\underline{9}-\underline{11}]$ can be achieved by using PhCs.

Recently, new spatial effects in light beam propagation were introduced by considering similar artificial materials: gain-loss-modulated materials (GLMMs) [12,13]. Such materials not only provide anomalous spatial dispersion, i.e., distortion of the isofrequency contours in wave-vector space, but also sharp peaks or valleys in the imaginary part of wave vectors, indicating a strong dependence of gain or losses on the direction of propagation. The regions of anisotropic gain or loss lie near the edges of the first Brillouin zone (BZ) and give rise to a strong dependence of gain on the direction of propagation [13]. Hence, GLMMs introduce new diffusive properties, while also modifying the spatial dispersion relations and diffractive properties. Therefore, 2D GLMMs also allow beam shaping effects that can be used, among other functions, for spatial filtering purposes, as has been demonstrated for loss-modulated materials (LMMs) [14] and broad-area semiconductor amplifiers $[\underline{15}, \underline{16}]$. Also $\overline{1 \mathrm{D}}$ gainloss layered structures have been considered for imaging purposes [17-20] or in the context of parity-time symmetry [21].

The present paper is devoted to analyzing the diffraction management associated with 2D LMMs and to studying the possibility of obtaining focusing, insensitive to alignment, from such structures. We consider LMMs to characterize the spatial propagation effects related to gain-loss modulation in order to avoid saturation and nonlinear effects introduced by a positive effective gain. Flat lensing is accounted for by convex-curved isofrequency contours of propagating modes in the wave-vector space. The effect arises because convex phase shifts are accumulated through either a negative-index material [22] or a $\mathrm{PhC}$ slab [무요 and are compensated by normal diffraction beyond the structure, determining the focalization distance from the flat lens. The ability to focus light by using flat surfaces is, however, not restricted to left-handed index materials or PhCs [23-26]. We demonstrate a significant localization of a light beam on propagation through a periodic structure with no index contrast but with losses modulated on the wavelength scale. We use a coupled mode expansion of Maxwell equations to analytically localize the parameter range that shows flat lensing for three different geometries. Moreover, numerical simulations of beam propagation performed by solving the full set of Maxwell equations using a finite-difference time-domain method confirm the predicted effect.

\section{COUPLED MODE EXPANSION}

We start from the wave equation for a monochromatic wave, which can be written in the single form:

$$
\nabla^{2} \vec{E}+n^{2} \frac{\omega^{2}}{c^{2}} \vec{E}=0
$$

We consider a 2D sinusoidal modulation of the complex refractive index, such as

$$
n(\vec{r})=n_{0}+2 n_{1}\left[\cos \left(\vec{q}_{1,0} \cdot \vec{r}\right)+\cos \left(\vec{q}_{0,1} \cdot \vec{r}\right)\right]
$$


where $n_{0}=1+i n_{0}^{\prime \prime}$ is a homogenous complex refractive index and $n_{1}=i n_{1}^{\prime \prime}$ is the amplitude of the modulation. The geometry is fixed by the two reciprocal lattice vectors $\vec{q}_{1,0}$ and $\quad \vec{q}_{0,1}$, where $\quad \vec{q}_{1,0}=q(\sin \theta, \cos \theta)$ and $\vec{q}_{0,1}=$ $q(-\sin \theta, \cos \theta)$. We assume a linearly polarized electric field perpendicular to the plane of the modulations, and we expand the electric field in terms of the harmonics of the modulation:

$$
E=\sum_{l, p} A_{l, p} e^{i \vec{k}_{l, p} \vec{r}}+\text { c.c. }
$$

where $\vec{k}_{l, p}=\vec{k}+l \vec{q}_{10}+p \vec{q}_{01}$. Next we substitute Eqs. (1b) and (2) into Eq. (1a) and obtain

$$
\sum_{l, p}\left[\nabla^{2} A_{l, p}+2 i \vec{k}_{l, p} \cdot \vec{\nabla} A_{l, p}-k_{l, p}^{2}+n^{2} k_{0}^{2} A_{l, p}\right] e^{i \vec{k}_{l, p} \cdot \vec{r}}=0,
$$

where $A_{l, p}$ stands for the amplitudes of each mode in the field expansion, and $k_{0}=2 \pi / \lambda$ is the wave vector in vacuum. The sinusoidal modulation couples only the neighboring modes. The coupling can be obtained from Eq. (3) and is expressed in a general form for the mode $(l, p)$ as

$$
\begin{aligned}
& {\left[k_{0}^{2}\left(n_{0}^{2}+4 n_{1}^{2}\right)-k^{2}-2 \vec{k}_{l, p} \cdot \vec{k}-k_{l, p}^{2}\right] A_{l, p}} \\
& \quad+2 n_{0} n_{1} k_{0}^{2}\left(A_{l+1, p}+A_{l-1, p}+A_{l, p+1}+A_{l, p-1}\right) \\
& \quad+n_{1}^{2} k_{0}^{2}\left(A_{l+2, p}+A_{l-2, p}+A_{l, p+2}+A_{l, p-2}\right) \\
& \quad+2 n_{1}^{2} k_{0}^{2}\left(A_{l+1, p+1}+A_{l+1, p-1}+A_{l-1, p+1}+A_{l-1, p-1}\right)=0 .
\end{aligned}
$$

The modulation lattice is characterized by the reciprocal lattice vectors and the angle between them. We consider three general geometries with obtuse, acute, and right angles between lattice vectors and assume an overall lossy material with $n_{0}^{\prime \prime}=0.2$ and a loss modulation $n_{1}^{\prime \prime}=0.095$. We shall refer to the nonorthogonal case as a rhombic lattice, where we consider propagation along both the long diagonal (ГM crystallographic direction) and the short diagonal ( $\Gamma K$ crystallographic direction). For these two cases the system can be described by using only three modes in the above developed expansion, since other modes are weakly excited by the entering field for the considered range of frequencies. These three modes are $(l, p)=(0,0),(0,-1)$, and $(-1,0)$, which, in the absence of coupling (homogenous material), correspond to three perfect circles in the reciprocal (wave-vector) space. At resonance, the three circles intersect at the edge of the first BZ. For the square lattice, however, owing to the structural symmetry, four circles intersect at resonance, and hence at least four modes have to be considered to describe the system, namely, $(l, p)=(0,0),(0,-1),(-1,0)$, and $(-1,-1)$. The modes of Eqs. (3) and (4) are similar to those of the standard plane wave expansion method for PhCs; however, in this case [the $k(\omega)$ approach] we fix the frequency to be real values to calculate the complex-valued propagation wavenumber, $k_{z}$.

Here, the system of coupled Eqs. (2), for the considered modes, provides complex eigenmodes for the wave vector $\vec{k}$. The real part of such eigenvalues describes the spatial dispersion curves, while the imaginary part corresponds to attenuation. Without loss of generality, we consider the angle between the reciprocal lattice vectors, $2 \theta=105^{\circ}$ and $2 \theta=75^{\circ}$ for the rhombic case $\left(2 \theta=90^{\circ}\right.$ for a square lattice). Figures $1(\mathrm{a})$ and $1(\mathrm{~b})$ show the calculated spatial dispersion
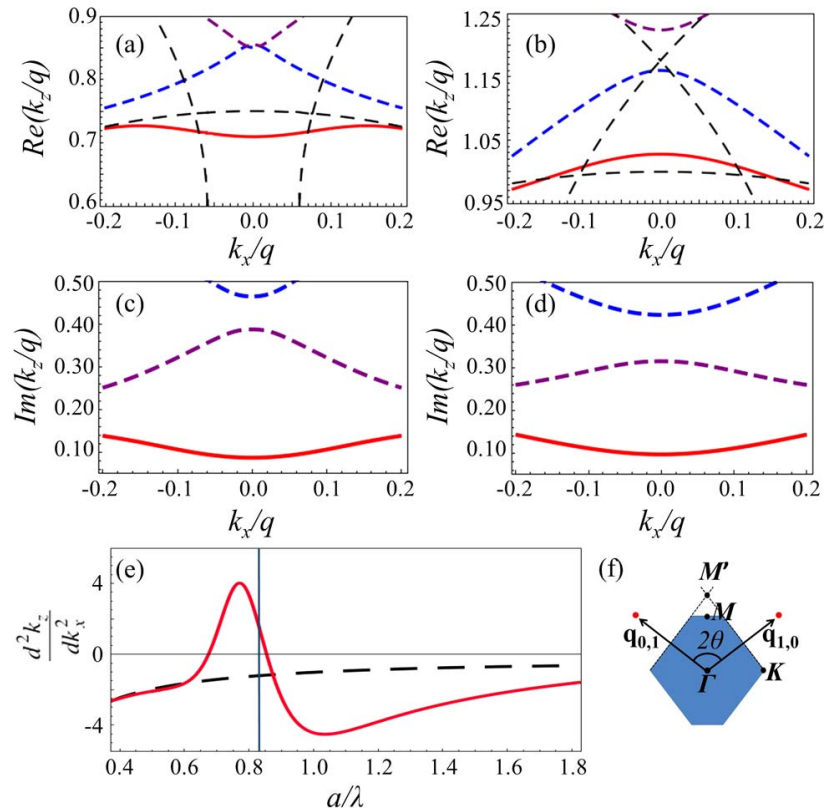

Fig. 1. Real spatial dispersion curves of the first three modes [real part of $k_{z}$ as a function of $k_{x}$, where $\left.\vec{k}=\left(k_{x}, k_{z}\right)\right]$ in normalized $q$ units, for (a) $a / \lambda=0.76$ and (b) $a / \lambda=1.0$, just below and above the $M^{\prime}$ point lying at $a / \lambda=0.83$. (c), (d) Attenuation, imaginary part of $k_{z}$ as a function of $k_{x}$, in normalized $q$ units for the same modes. The curvature of the dispersion, for the least attenuated mode at $k_{x}=0$, is shown depending on frequency (in normalized $a / \lambda$ units) for propagation along the diagonal for $2 \theta=75^{\circ}$. The black dashed lines indicate the dispersion for homogenous propagation in (a) and (b) and the corresponding curvatures in (e). The first BZ (blue area) and the corresponding symmetry points are represented in (f).

curves for the first case (propagation along $\Gamma M$ ) for two given frequencies, lying just higher and lower than the $M^{\prime}$ point; Figs. 1(c) and 1(d) depict the attenuation of each corresponding mode. To determine the character of diffraction, we shall consider the dominant mode, i.e., the less attenuated one (with a smaller imaginary component). Inspecting the curve for the diffraction of such a less attenuated mode for both frequencies, we observe that it has different signs for different frequencies. For a frequency lying just below the $M^{\prime}$ point, the curvature is convex (positive), indicating negative diffraction; see the solid curve in Fig. 1(a). However, the curvature becomes strongly negative (positive diffraction) for higher frequencies; see Fig. 1(b). We analyze the diffraction dependence of frequency through the second derivative of the in-axis spatial longitudinal dispersion, $\partial^{2} k_{z} / \partial k_{x}^{2}$, at $k_{x}=0$, which is depicted by the solid red line in Fig. 1(e), and we compared it to normal diffraction in free space (dashed black line). As expected, for small frequencies the beam suffers from normal diffraction, and therefore the curvature is negative. What is more important, we observe negative diffraction just below the $M^{\prime}$ point. With further increases in frequency, the curvature becomes strongly negative (diffraction is positive and much stronger than in homogenous space), and, finally, the curvature increases, tending to zero. [It has to be noted that for the case of propagation along the long diagonal, the resonance occurs at $M^{\prime}$ rather than the edge of first BZ $(M)$ as shown in Fig. 1(f)].

Similar behavior can be observed for propagation along $\Gamma K$; see Fig. 2(a). Also, for a square lattice, the four-mode analysis provides analogous results, as shown in Fig. 2(c). 
(a)



(b)

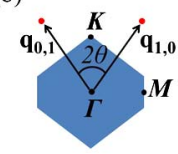

(c)

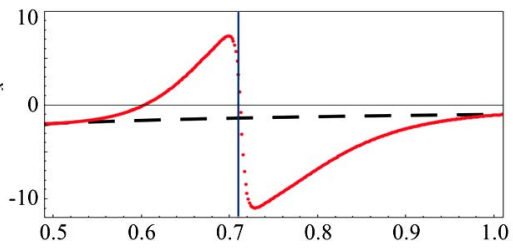

(d)

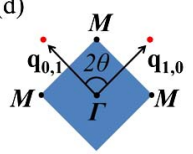

Fig. 2. Curvature of the dispersion, for the least attenuated mode at $k_{x}=0$, is shown depending on frequency (in normalized $a / \lambda$ units) for propagation along the diagonal (a) for $2 \theta=105^{\circ}$ and (c) $2 \theta=90^{\circ}$. The black dashed lines in (a) and (c) denote the curvature of dispersion for homogenous propagation. (b), (c) Corresponding reciprocal lattice and symmetry points.

In both cases, negative diffraction occurs for different frequencies, all lying just below the corresponding first BZ edge. Hence the predicted effect of negative diffraction is expected to be general for any rhombic structure. In all cases, such negative diffraction is expected to lead to focalization of a beam propagating beyond such a structure. Note that in this case resonance occurs precisely at the edge of the first BZ.

\section{NUMERICAL ANALYSIS}

In calculations we consider a finite-sized $2 \mathrm{D}$ periodic structure with flat interfaces made of absorbing cylinders embedded in an inactive background. The real refractive index contrast is considered to be zero; thus all observed effects will be directly associated with the loss modulation. In a realistic system one should take into account both index and loss modulation. The radius of the cylinders is fixed to be $R=0.2 a$, where $a$ denotes the lattice constant of the structure (being $a=2 \pi / q)$. The absorption coefficient is $\alpha=$ $5 \times 10^{4} \mathrm{~cm}^{-1}$ (for $\lambda=1 \mu \mathrm{m}$ ), which corresponds to a complex valued refractive index of $n_{\text {cyl }}=1+0.4 i$ for the lossy cylinders. We consider the same geometries as in the previous analytic study, namely, $2 \theta=105^{\circ}, 75^{\circ}, 90^{\circ}$. To perform the numerical analysis, we propagate a Gaussian beam with a width of $1.5 \mu \mathrm{m}$, and an electric field linearly polarized parallel to the rods, through a 15-period-long structure, using the commercial finite-difference time-domain algorithm CrystalWave [27]. The total simulation area is $60 \mu \mathrm{m}$ (transverse) $\times 100 \mu \mathrm{m}$ (longitudinal) with a grid square cell of length $0.0475 \mu \mathrm{m}$, and the time step is fixed to provide numerical stability. Reflections at boundaries are avoided by using perfectly matched layers boundary conditions. The on-axis transmitted intensity is analyzed as a function of the carrier frequency of the incident beam, and, as a reference for comparison, we also calculate the propagation of the input beam through a homogenous material (air).

Figure 3 summarizes the calculations in the case of a rhombic lattice and propagation along the long diagonal ( $\Gamma M$ crystallographic direction). The transmitted intensity, plotted as a function of the beam carrier frequency, exhibits a hightransmission window on either side of the high-symmetry $M^{\prime}$ point corresponding to $a / \lambda=0.83$. It is important to note

that such a high-transmission band, determined for a LMM, lies precisely where a low-intensity band or bandgap would be expected in the case of a PhC slab, i.e., in the case where the real part of the refractive index would be modulated instead of the imaginary one. Inspecting a cross section of such an intensity map, shown in Figs. $\underline{3(b)}$ and $\underline{3(\mathrm{c})}$, at two given distances from the sample, we observe the expected focalization because of the negative diffraction inside the structure. It is clear from these figures that the beam emerging from a LMM has a narrower profile than the reference beam. Hence, on incidence of a linearly polarized Gaussian beam, the structure provides focalization beyond it.

To demonstrate the flat lensing effect and to allow some comparison with the analytic predictions, we also consider the phase profiles of the beam beyond the LMM crystal. We obtain the phases of the spatial Fourier components (far field) of the exiting beam just beyond the structure and calculate its curvature (second derivative in wave-vector space). The dependence of phase curvature on frequency is reproduced from the numerical analysis in Fig. 4 . Note that in this case we obtain the phase curvature on a plane parallel to the exiting facet, on which normal diffraction would lead to an opposite curvature. Hence, in order to make the comparison with analytics (where normal diffraction provides a positive curvature), we reverse the sign of the curvature obtained from the numeric propagation. We see that, in good agreement with Fig. 1(e), negative diffraction occurs behind the $M^{\prime}$ point. We note that we compare the numerical simulations with our previous analytic results on a simple sinusoidal modulation of the refractive index. For the considered frequencies light propagation is especially sensitive to the periodicity of the
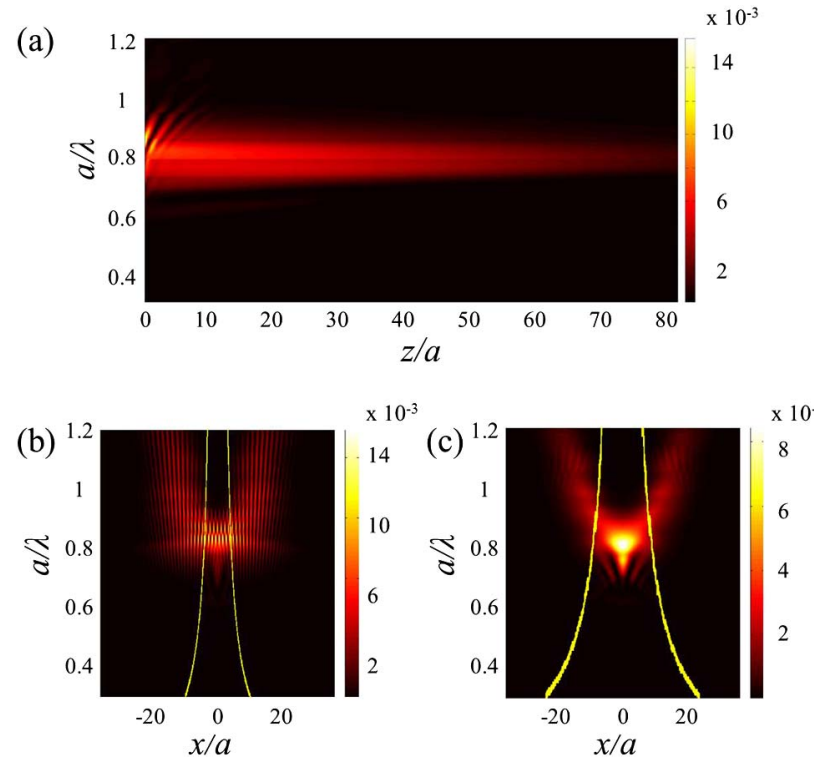

Fig. 3. Beam propagation through the long diagonal of a LMM rhombic structure, made of lossy cylinders embedded in air, $R=0.2 a$, where $a$ is the direct lattice distance, $a=2 \pi / q$. (a) Finite-difference time domain transmission map for cylinders with $n_{\text {cyl }}=1+0.4 i$; the vertical axis denotes the carrier frequency, in $a / \lambda$ units, of the $1.5 \mu \mathrm{m}$ wide incident Gaussian and the horizontal axis, the normalized distance from the sample. (b), (c) Transverse cross section at the output face of the device and at a distance of $z=36 a$ after the structure, denoted D1 and D2 in (a), where the continuous yellow curve is for comparison with propagation in free space. 



Fig. 4. (a) Curvature of the phase of the spatial Fourier transform of a Gaussian beam just exiting the LMM slab. (b), (c), (d) Comparison of the phase profile and the same phase profile for a beam propagating in free space (dotted curve), for $a / \lambda=0.67,0.81$, and 0.97 , respectively.

modulation (or to the first spatial harmonics of the modulation) rather than to a specific distribution within the unit cell.

Figures $\underline{4(\mathrm{~b})}, \underline{4(\mathrm{c})}$, and $\underline{4(\mathrm{~d})}$ show the phase profile as a function of the wave-vector component perpendicular to the direction of propagation, for three different frequencies, compared to the same curvature in free-space propagation. We can observe that, for frequencies below the resonance

(a)



(b)
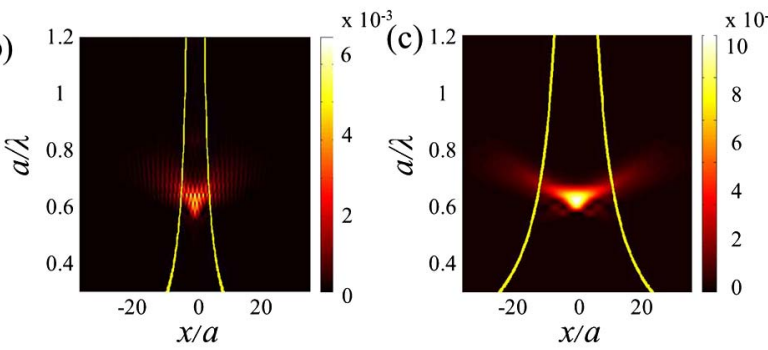

(d)

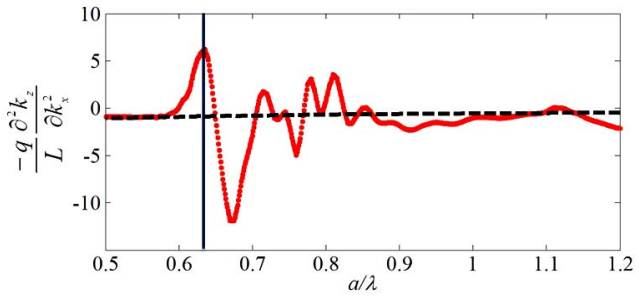

Fig. 5. Beam propagation through the short diagonal of a LMM rhombic structure. (a) Transmission map at $x / a=0$ and (b), (c) transverse cross section at D1 and D2. (d) Phase curvature of the beam exiting after propagation through the LMM. frequency, the curvature resembles curvature in free space [Fig. 4(b)]; it becomes strongly convex just below the $M^{\prime}$ point [see Fig. 4(c)]. Just above the $M^{\prime}$ point it becomes strongly negative, as shown in Fig. 4(d). In addition, for frequencies close to resonance, the spatial components around $k_{x}=0$ have near-zero attenuation. Hence, for such frequencies the structure becomes less lossy; this, combined with a positive curvature of the phase, results in a less attenuated (highly transmitted), focalized beam beyond the LMM. While the maximum intensity at the focal point is around $10 \%$ of that of the reference beam, it is 3 orders of magnitude higher when compared to propagation through a homogenous lossy medium with the same averaged loss.

To show that this behavior could be generic for all geometries in LMM, we extend our numerical calculations to propagation along the short diagonal of a rhombic lattice and also a square structure. Also, in these cases a high-transmission window is found at the expected frequencies, by the BZ edge, lying at $a / \lambda=0.63$ in Fig. $\underline{5(\mathrm{a})}$ and at $a / \lambda=0.71$ in Fig. $\underline{6}$. Also, in Figs. 5(c), 5(d), 6(c), and 6(d) the narrowing of the exiting beam is clearly visible compared to the reference. In all cases, on propagation inside the LMM, the beam acquires a change in the curvature of the phase. For frequencies below the highsymmetry point, the curvature becomes positive. According to numerical simulations and analytics, the effect becomes stronger for the square lattice, when four modes come into play [compare Figs. 6(d) with $5(\mathrm{~d})$ and $4(\mathrm{a})$ for numerics, and Fig. $\underline{1(\mathrm{~g})}$ with $\underline{1(\mathrm{f})}$ and $\underline{1(\mathrm{e})}$ for analytics].

(a)

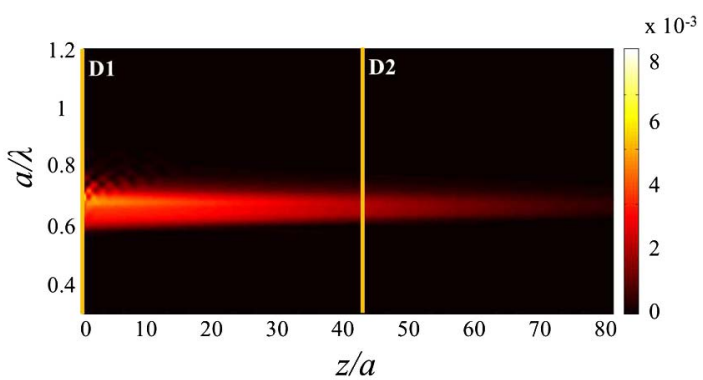

(b)
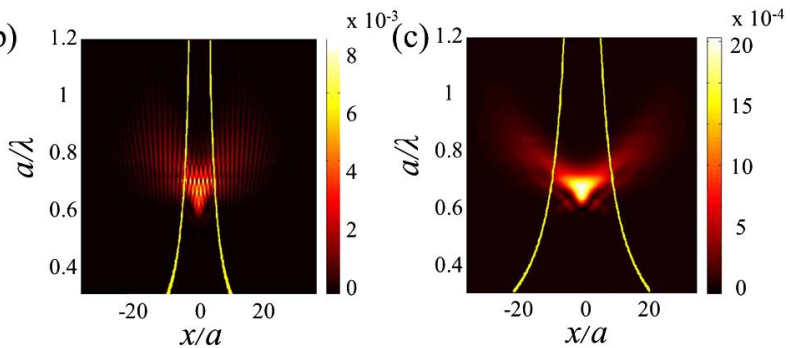

(d)

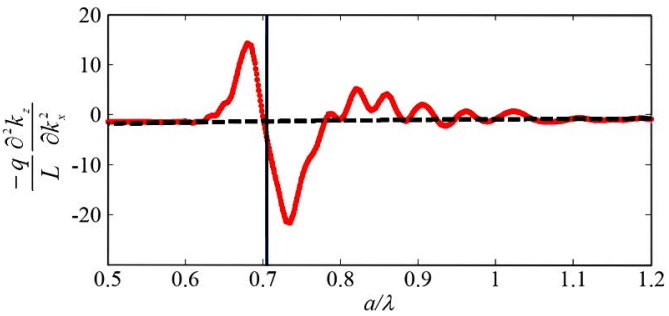

Fig. 6. Beam propagation through the diagonal of a LMM square structure. (a) Transmission map at $x / a=0$. (b), (c) Transverse cross section at D1 and D2. (d) Phase curvature of the beam exiting after propagation through the LMM. 


\section{CONCLUSION}

To conclude, we show that LMM materials with periodic modulations on the wavelength scale can lead to a substantial focalization of a beam propagating through a short slab with flat-flat interfaces. We have considered three different structural geometries, corresponding to propagation along the diagonals of a rhombic and square lattice, and in all cases find analogous results. The light intensity map of a Gaussian beam exiting the structure exhibits a high-transmission window for frequencies close to the edges of the first BZ, which results from the anisotropic attenuation provided by the periodicity. For LMM, such high transmittance occurs precisely at frequencies where a low transmission or bandgap open in the case of PhCs. The focusing after the crystal slab with a thickness of ten wavelengths is confirmed by a deeper analysis of the phase profile. For given frequencies, the phase curvature becomes positive, indicating negative diffraction, which provides focalization beyond the structure. Moreover, the numerically obtained curvature follows the analytical estimations based on the second derivative of the spatial dispersion diagrams. The control of light beam propagation by miniaturized devices is important not only from a fundamental point of view but also as an actual requirement for applications. The predicted phenomenon is expected to be generic for spatially modulated materials and other kinds of waves, such as, for instance, acoustic waves in lossy sonic crystals.

\section{REFERENCES}

1. L. Jia and E. L. Thomas, "Two-pattern compound photonic crystals with a large complete photonic band gap," Phys. Rev. A 84, 033810 (2011).

2. L. Jia and E. L. Thomas, "Theoretical study on photonic devices based on a commensurate two-pattern photonic crystal," Opt. Lett. 36, 3416-3418 (2011).

3. H. Kosaka, T. Kawashima, A. Tomita, M. Notomi, T. Tamamura, T. Sato, and S. Kawakami, "Self-collimating phenomena in photonic crystals," Appl. Phys. Lett 74, 1212-1214 (1999).

4. K. Staliunas and R. Herrero, "Nondiffractive propagation of light in photonic crystals," Phys. Rev. E 73, 016601 (2006).

5. D. Chigrin, S. Enoch, C. S. Torres, and G. Tayeb, "Self-guiding in two-dimensional photonic crystals," Opt. Express 11, 1203-1211 (2003).

6. C. Luo, S. G. Johnson, J. D. Joannopoulos, and J. B. Pendry, "All-angle negative refraction without negative effective index," Phys. Rev. B 65, 201104 (2002).

7. E. Cubukcu, K. Aydin, E. Ozbay, S. Foteinopoulou, and C. M. Soukoulis, "Electromagnetic waves: negative refraction by photonic crystals," Nature 423, 604-605 (2003).

8. A. Berrier, M. Mulot, M. Swillo, M. Qiu, L. Thylen, A. Talneau, and S. Anand, "Negative refraction at infrared wavelengths in a two-dimensional photonic crystal," Phys. Rev. Lett. 93, 073902 (2004).
9. L. Maigyte, T. Gertus, M. Peckus, J. Trull, C. Cojocaru, V. Sirutkaitis, and K. Staliunas, "Signatures of light-beam spatial filtering in a three-dimensional photonic crystal," Phys. Rev. A 82, 043819 (2010).

10. E. Colak, A. O. Cakmak, A. E. Serebryannikov, and E. Ozbay, "Spatial filtering using dielectric photonic crystals at beam-type excitation,” J. Appl. Phys. 108, 113106 (2010).

11. K. Staliunas and V. J. Sánchez-Morcillo, "Spatial filtering of light by chirped photonic crystals," Phys. Rev. A 79, 053807 (2009).

12. K. Staliunas, R. Herrero, and R. Vilaseca, "Subdiffraction and spatial filtering due to periodic spatial modulation of the gain-loss profile,” Phys. Rev. A 80, 013821 (2009).

13. M. Botey, R. Herrero, and K. Staliunas, "Light in materials with periodic gain-loss modulation on a wavelength scale," Phys. Rev. A 82, 013828 (2010).

14. N. Kumar, M. Botey, R. Herrero, Y. Loiko, and K. Staliunas, "High-directional wave propagation in periodic loss modulated materials," Photon. Nanostr. Fundam. Appl. 10, 644-650 (2012).

15. R. Herrero, M. Botey, M. Radziunas, and K. Staliunas, "Beam shaping in spatially modulated broad-area semiconductor amplifiers," Opt. Lett. 37, 5253-5255 (2012).

16. M. Radziunas, M. Botey, R. Herrero, and K. Staliunas, "Intrinsic beam shaping mechanism in spatially modulated broad area semiconductor amplifiers," Appl. Phys. Lett (to be published).

17. S. A. Ramakrishna and J. B. Pendry, "Removal of absorption and increase in resolution in a near-field lens via optical gain," Phys. Rev. B 67, 201101 (2003).

18. M. A. Vincenti, D. de Ceglia, V. Rondinone, A. Ladisa, A. D’Orazio, M. J. Bloemer, and M. Scalora, "Loss compensation in metal-dielectric structures in negative-refraction and super-resolving regimes,” Phys. Rev. A 80, 053807 (2009).

19. P. A. Belov and Y. Hao, "Subwavelength imaging at optical frequencies using a transmission device formed by a periodic layered metal-dielectric structure operating in the canalization regime," Phys. Rev. B 73, 113110 (2006).

20. D. de Ceglia, M. A. Vincenti, M. G. Cappeddu, M. Centini, N. Akozbek, A. D’Orazio, J. W. Haus, M. J. Bloemer, and M. Scalora, "Tailoring metallodielectric structures for superresolution and superguiding applications in the visible and near-IR ranges," Phys. Rev. A 77, 033848 (2008).

21. K. G. Makris, R. El-Ganainy, D. N. Christodoulides, and Z. H. Musslimani, "Beam dynamics in PT symmetric optical lattices," Phys. Rev. Lett. 100, 103904 (2008).

22. J. B. Pendry, "Negative refraction makes a perfect lens," Phys. Rev. Lett. 85, 3966-3969 (2000).

23. V. G. Veselago, "The electrodynamics of substances with simultaneously negative values of $\varepsilon$ and $\mu$," Sov. Phys. Usp. 10, 509 (1968).

24. P. V. Parimi, W. T. Lu, P. Vodo, and S. Sridhar, "Imaging by flat lens using negative refraction," Nature 426, 404 (2003).

25. N. Fang, H. Lee, C. Sun, and X. Zhang, "Subdiffractionlimited optical imaging with a silver superlens," Science 308, 534-537 (2005).

26. H. Lee, Y. Xiong, N. Fang, W. Srituravanich, S. Durant, M. Ambati, C. Sun, and X. Zhang, "Realization of optical superlens imaging below the diffraction limit," New J. Phys. 7, 255 (2005).

27. CrystalWave software by Photon Design Ltd., http://www .photond.com. 